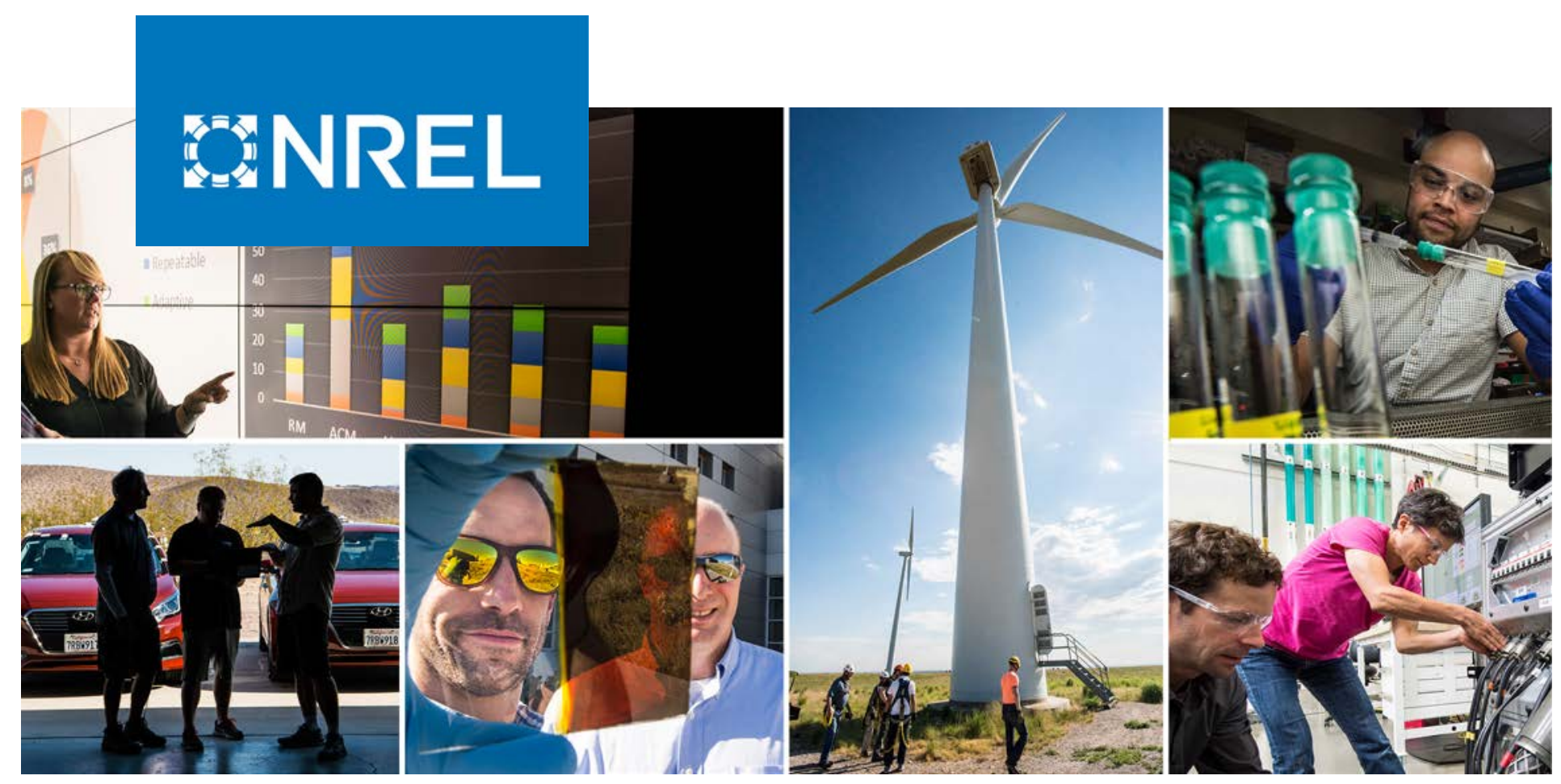

\title{
Comparative Small-Signal Stability Analysis of Grid-Forming and Grid- Following Inverters in Low-Inertia Power Systems
}

\section{Preprint}

Lizhi Ding, ${ }^{1,2}$ Xiaonan $\mathrm{Lu}^{2}$ and Jin Tan ${ }^{1}$

1 National Renewable Energy Laboratory

2 Temple University

Presented at the 47th Annual Conference of the IEEE Industrial Electronics Society (IECON)

October 13-16, 2021

NREL is a national laboratory of the U.S. Department of Energy Office of Energy Efficiency \& Renewable Energy

Operated by the Alliance for Sustainable Energy, LLC

This report is available at no cost from the National Renewable Energy Laboratory (NREL) at www.nrel.gov/publications.
Conference Paper

NREL/CP-5C00-80335

January 2022 


\title{
GNREL
}

\section{Comparative Small-Signal Stability Analysis of Grid-Forming and Grid- Following Inverters in Low-Inertia Power Systems}

\section{Preprint}

\author{
Lizhi Ding, ${ }^{1,2}$ Xiaonan Lu, ${ }^{2}$ and Jin $\operatorname{Tan}^{1}$ \\ 1 National Renewable Energy Laboratory \\ 2 Temple University
}

\author{
Suggested Citation \\ Ding, Lizhi, Xiaonan Lu, and Jin Tan. 2022. Comparative Small-Signal Stability Analysis \\ of Grid-Forming and Grid-Following Inverters in Low-Inertia Power Systems: Preprint. \\ Golden, CO: National Renewable Energy Laboratory. NREL/CP-5C00-80335. \\ https://www.nrel.gov/docs/fy22osti/80335.pdf.
}

(c) 2022 IEEE. Personal use of this material is permitted. Permission from IEEE must be obtained for all other uses, in any current or future media, including reprinting/republishing this material for advertising or promotional purposes, creating new collective works, for resale or redistribution to servers or lists, or reuse of any copyrighted component of this work in other works.

NREL is a national laboratory of the U.S. Department of Energy Office of Energy Efficiency \& Renewable Energy Operated by the Alliance for Sustainable Energy, LLC

This report is available at no cost from the National Renewable Energy Laboratory (NREL) at www.nrel.gov/publications.

Contract No. DE-AC36-08GO28308
Conference Paper

NREL/CP-5C00-80335

January 2022

National Renewable Energy Laboratory 15013 Denver West Parkway Golden, CO 80401

303-275-3000 • www.nrel.gov 


\section{NOTICE}

This work was authored in part by the National Renewable Energy Laboratory, operated by Alliance for Sustainable Energy, LLC, for the U.S. Department of Energy (DOE) under Contract No. DE-AC36-08GO28308. Funding provided by U.S. Department of Energy Office of Energy Efficiency and Renewable Energy Solar Energy Technologies Office Award Numbers 34224 and 37772. The views expressed herein do not necessarily represent the views of the DOE or the U.S. Government.

This report is available at no cost from the National Renewable Energy Laboratory (NREL) at www.nrel.gov/publications.

U.S. Department of Energy (DOE) reports produced after 1991 and a growing number of pre-1991 documents are available free via www.OSTI.gov.

Cover Photos by Dennis Schroeder: (clockwise, left to right) NREL 51934, NREL 45897, NREL 42160, NREL 45891, NREL 48097, NREL 46526.

NREL prints on paper that contains recycled content. 


\title{
Comparative Small-Signal Stability Analysis of Grid- Forming and Grid-Following Inverters in Low-Inertia Power Systems
}

\author{
Lizhi Ding ${ }^{1,2}$, Student Member, IEEE, Xiaonan Lu $^{2}$, Member, IEEE, Jin Tan* ${ }^{1}$, Member, IEEE \\ 1. Power Systems Engineering Center, NREL, Golden, CO, USA 2. College of Engineering, Temple University, Philadelphia, PA, USA
}

\begin{abstract}
Inverter-based resources (IBRs) are gradually replacing conventional fossil energy in modern power systems, which could make the systems susceptible as a result of insufficient inertia, especially when moving towards a $100 \%$ inverter-based power grid. Grid-following (GFL) control is a common gridinteractive inverter control algorithm, while grid-forming (GFM) control as an emerging inverter control function has drawn increasing awareness nowadays. Further, the corresponding stability issues along with a comparative study on low-inertia power systems should still be investigated. This paper focuses on small-signal stability and conducts a comprehensive analysis of GFM and GFL controls, with emphasis on the interactions between synchronous generators (SGs) and IBRs. The detailed modelings of GFM and GFL inverters as well as SGs are established. The coupling mechanism of IBRs and SGs is then conducted through participation factor analysis. Finally, the impacts of critical parameters on system stability are identified. The theoretical analyses are validated through time-domain simulations in MATLAB/Simulink.
\end{abstract}

Keywords-grid-following, grid-forming, modal analysis, participation factor, small-signal stability, synchronous generator

\section{INTRODUCTION}

The increasing penetration of renewable energy resources poses significant challenges in modern power systems in terms of dynamic modeling and control, active stabilization, and others [1]. With the integration of inverter-based resources (IBRs), such as solar and wind generation units and battery energy storage systems, conventional synchronous generators (SGs) are being gradually replaced, and the power grid is moving toward a low-inertia system. Comprehensive and accurate modeling of the low-inertia power system is urgently needed to provide potential solutions to the emerging problems.

Small-signal stability analysis for SGs is well established; however, the stator and network dynamics are usually neglected in conventional power system stability analysis [2]. With the increasing integration of IBRs, there is tremendous interest in $100 \%$ IBR penetration [3]-[5]. In particular, it was revealed in [3] that for power-electronics-dominated power systems, stability issues can result from the coupling of multi-timescale control loops, and the interactions of multiple inverters and other power devices; however, this analysis was limited to $100 \%$ inverter-based microgrids, and the scalability should be further verified. The impact of the dynamic behavior of photovoltaic (PV) power generation systems on the short-term transmission system voltage stability was analyzed in [4], whereas the results were derived based on the simplified and stiff grids. An approach to analyze the impact of increased penetration of doubly-fed induction generator wind turbines on the transient and small-signal stability of a large power system was developed in [5]; however, the inner control loops of the inverter-interfaced wind turbines are neglected, and some critical oscillation modes could be missing.

The existing grid-interactive inverters are generally operated in grid-following (GFL) control mode [6]. The output power is regulated by measuring the grid voltage phase angle through dedicated phase-lock loops (PLLs). Although frequency-watt control could be developed for GFL inverters to support fast grid frequency regulation [7], it cannot actively establish grid frequency when transitioning to islanded operation. In contrast, grid-forming (GFM) inverters can actively establish frequency and voltage at the local inverter level, which makes them widely used in microgrids and external large-scale distribution and even transmission systems [8]. The comparative study between GFM and GFL inverter can be found in [9], but the inverter and SG models are reduced-order models. Further, the interactions between inverters and conventional SGs should be explored.

This paper investigates the coupling mechanism between inverters (with either GFL or GFM control) and SGs through modal analysis. The GFM and GFL inverters share some common oscillation modes while also featuring unique modes due to the interactions among different control loops. Further, this paper discusses and verifies the relationship between IBR penetration level and small-signal stability constraints.

The remainder of this paper is structured as follows. Section II presents the detailed dynamic modeling of SGs and GFL as well as GFM inverters to derive a holistic model of the entire system. Subsequently, Section III conducts a comparative study among various inverter control diagrams with modal analysis and participation factor analysis. Section IV analyzes and validates the impacts of key parameters (e.g., the length of the transmission line and penetration level, etc.) on system smallsignal stability through time-domain tests. Section V draws the conclusions and discusses future work.

\section{SMALL-SIGNAL MODELING OF LOW-INERTIA POWER GRIDS \\ WITH SYNCHRONOUS GENERATORS AND INVERTERS}

Without loss of generality and to highlight the impacts on the system stability of IBRs, Fig. 1 shows a simplified two-terminal system architecture. In particular, all the SGs are aggregated into a single SG and all IBRs are aggregated into one inverter. Meanwhile, one remote load center connected at Bus \#2 and two local loads connected at Bus \#1 and Bus \#3 are also considered. The location of the load center can be adjusted with varying lengths of the SG-side transmission line and the inverter-side transmission line.

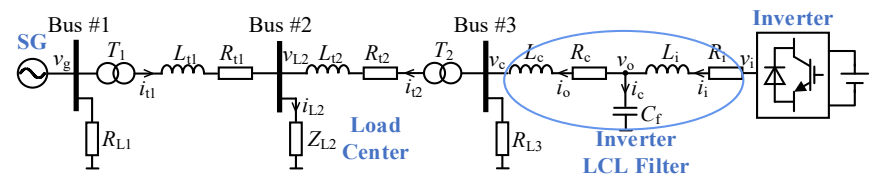

Fig. 1. One-line system diagram with aggregated SG and IBR. 


\section{A. Modeling the Synchronous Generator}

A traditional salient-pole type $\mathrm{SG}$ is considered for this study with its governor, turbine, and exciter, as shown in Fig 2. The dynamics of the stator, exciter, and $q$-axis damping circuit are considered and the models of the exciter and turbine are simplified to first-order models. Note that all the quantities are converted to the per-unit system based on the reciprocal per-unit system presented in [2].

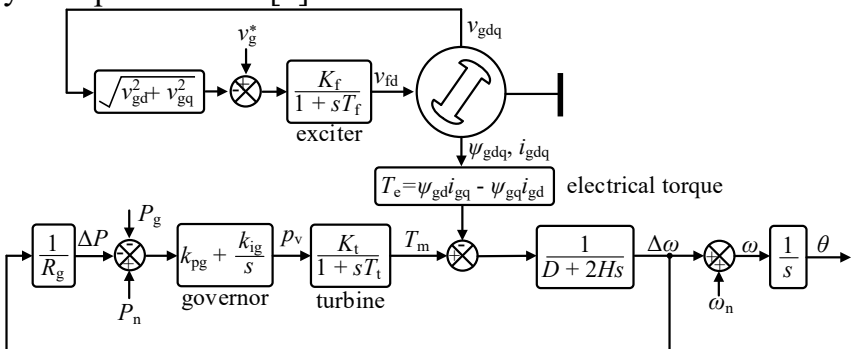

Fig. 2. Block diagram of SG.

With the voltage relationship and flux linkage relationship in [2], the small-signal representations of $\psi_{\mathrm{d}}, \psi_{\mathrm{q}}, \psi_{\mathrm{fd}}, \psi_{\mathrm{kq}}$ are given: $\left[\begin{array}{c}\Delta \dot{\psi}_{\mathrm{d}} \\ \Delta \dot{\psi}_{\mathrm{q}} \\ \Delta \dot{\psi}_{\mathrm{fd}} \\ \Delta \dot{\psi}_{\mathrm{kq}}\end{array}\right]=-\mathbf{R}\left[\begin{array}{c}-\Delta i_{\mathrm{d}} \\ -\Delta i_{\mathrm{q}} \\ \Delta i_{\mathrm{fd}} \\ \Delta i_{\mathrm{kq}}\end{array}\right]-\mathbf{W}\left[\begin{array}{c}\Delta \psi_{\mathrm{d}} \\ \Delta \psi_{\mathrm{q}} \\ \Delta \psi_{\mathrm{fd}} \\ \Delta \psi_{\mathrm{kq}}\end{array}\right]+\left[\begin{array}{c}\omega_{\mathrm{b}} \psi_{\mathrm{q}} \\ -\omega_{\mathrm{b}} \psi_{\mathrm{d}} \\ 0 \\ 0\end{array}\right] \Delta \omega+\omega_{\mathrm{b}}\left[\begin{array}{c}\Delta v_{\mathrm{d}} \\ \Delta v_{\mathrm{q}} \\ \Delta v_{\mathrm{fd}} \\ \Delta v_{\mathrm{kq}}\end{array}\right],\left[\begin{array}{c}-\Delta i_{\mathrm{d}} \\ -\Delta i_{\mathrm{q}} \\ \Delta i_{\mathrm{fd}} \\ \Delta i_{\mathrm{kq}}\end{array}\right]=\mathbf{L}^{-1}\left[\begin{array}{c}\Delta \psi_{\mathrm{d}} \\ \Delta \psi_{\mathrm{q}} \\ \Delta \psi_{\mathrm{fd}} \\ \Delta \psi_{\mathrm{kq}}\end{array}\right]$ (1) where $\psi_{\mathrm{d}}, \psi_{\mathrm{q}}, \psi_{\mathrm{fd}}, \psi_{\mathrm{kq}}, v_{\mathrm{d}}, v_{\mathrm{q}}, v_{\mathrm{fd}}, v_{\mathrm{kq}} i_{\mathrm{d}}, i_{\mathrm{q}}, i_{\mathrm{fd}}, i_{\mathrm{kq}}$ are the flux linkage, voltage and current of a typical SG, respectively; $\mathbf{R}, \mathbf{W}$, $\mathbf{L}$ are the resistance, rotor speed, and inductance matrices, respectively, as derived in [2]; and $\omega_{\mathrm{b}}$ is the base value of the system frequency. Becasues of space constrains, $\mathbf{R}, \mathbf{W}$, and $\mathbf{L}$ are not detailed here.

The swing equations that describe the mechanical dynamics of the SG are as follows:

$$
\begin{gathered}
\dot{\delta}=\omega_{\mathrm{b}}\left(\omega-\omega_{\text {com }}\right) \rightarrow \Delta \dot{\delta}=\omega_{\mathrm{b}} \Delta \omega-\omega_{\mathrm{b}} \Delta \omega_{\text {com }} \\
2 H \dot{\omega}+D(\omega-1)=T_{\mathrm{m}}-T_{\mathrm{e}} \rightarrow \Delta \dot{\omega}=-\frac{D \Delta \omega}{2 H}+\frac{\Delta T_{\mathrm{m}}}{2 H}-\frac{\Delta T_{\mathrm{e}}}{2 H}
\end{gathered}
$$

where $D$ is the damping coefficient; $H$ is the inertia constant; $T_{\mathrm{m}}$ is the mechanical torque; $T_{\mathrm{e}}$ is the electrical torque; and $\delta$ is the phase angle of the SG with respect to the common reference frame with the angular frequency, $\omega_{\text {com }}$.

The first-order model is used to represent the turbine. For the governor, an intermediate variable, $\varepsilon_{\mathrm{v}}$, is used to model the proportional-integral (PI) governor. Hence, it yields:

$$
\begin{gathered}
\dot{\varepsilon}_{\mathrm{v}}=\left[P_{\mathrm{n}}-P_{\mathrm{g}}-(\omega-1) / R_{\mathrm{g}}\right] \rightarrow \Delta \dot{\varepsilon}_{\mathrm{v}}=-\Delta P_{\mathrm{g}}-\Delta \omega / R_{\mathrm{g}} \\
p_{\mathrm{v}}=\left(k_{\mathrm{pg}}+\frac{k_{\mathrm{ig}}}{S}\right) \dot{\varepsilon}_{\mathrm{v}} \rightarrow \Delta p_{\mathrm{v}}=-k_{\mathrm{pg}} \Delta P_{\mathrm{G}}-\frac{k_{\mathrm{pg}}}{R_{\mathrm{g}}} \Delta \omega+k_{\mathrm{ig}} \Delta \varepsilon_{\mathrm{v}} \\
T_{\mathrm{m}}=\frac{K_{\mathrm{t}}}{1+s T_{\mathrm{t}}} p_{\mathrm{v}} \rightarrow \Delta \dot{T}_{\mathrm{m}}=-\frac{\Delta T_{\mathrm{m}}}{T_{\mathrm{t}}}+\frac{K_{\mathrm{t}}}{T_{\mathrm{t}}} \Delta p_{\mathrm{v}}
\end{gathered}
$$

where $R_{\mathrm{g}}$ is the droop coefficient; $P_{\mathrm{g}}$ is the output active power of SG; $P_{\mathrm{n}}$ is the setpoint; $T_{\mathrm{t}}$ is the turbine time constant; $K_{\mathrm{g}}$ is the turbine first-order model gain; and $p_{\mathrm{v}}$ is the valve position.

The exciter is also represented by a first-order model. Hence,

$$
v_{\mathrm{fd}}=\frac{K_{\mathrm{f}}}{1+s T_{\mathrm{f}}}\left(v_{\mathrm{g}}^{*}-v_{\mathrm{g}}\right) \rightarrow \Delta \dot{v}_{\mathrm{fd}}=-\frac{1}{T_{\mathrm{f}}} \Delta v_{\mathrm{fd}}-\frac{K_{\mathrm{f}}}{T_{\mathrm{f}}} \Delta v_{\mathrm{g}}
$$

where $T_{\mathrm{f}}$ is the exciter time constant; $K_{\mathrm{g}}$ is the exciter first-order model gain; and $v_{\mathrm{g}}$ is the $\mathrm{SG}$ output voltage.

The output current and the voltage of the SG should be converted into the common $D-Q$ frame to integrate it with the networks that are implemented in the common frame; thus, the following transformation should be conducted:

$$
i_{\mathrm{DQ}}=\mathbf{T} i_{\mathrm{dq}}, v_{\mathrm{dq}}=\mathbf{T}^{-1} v_{\mathrm{DQ}}
$$

where $\mathbf{T}=\left[\begin{array}{cc}\cos \delta & -\sin \delta \\ \sin \delta & \cos \delta\end{array}\right] ; \delta$ is the phase angle difference.

By linearizing these equations, the small-signal representations are obtained as:

$$
\begin{gathered}
\Delta \mathbf{I}_{\mathrm{DQ}}=\left[\begin{array}{c}
\Delta i_{\mathrm{D}} \\
\Delta i_{\mathrm{Q}}
\end{array}\right]=\mathbf{T}\left[\begin{array}{c}
\Delta i_{\mathrm{d}} \\
\Delta i_{\mathrm{q}}
\end{array}\right]+\mathbf{T}_{\mathrm{C}} \Delta \delta \\
\Delta \mathbf{V}_{\mathrm{dq}}=\left[\begin{array}{c}
\Delta v_{\mathrm{d}} \\
\Delta v_{\mathrm{q}}
\end{array}\right]=\mathbf{T}^{-1}\left[\begin{array}{c}
\Delta v_{\mathrm{D}} \\
\Delta v_{\mathrm{Q}}
\end{array}\right]+\mathbf{T}_{\mathrm{V}}^{-1} \Delta \delta \\
\text { where } \mathbf{T}_{\mathrm{C}}=\left[\begin{array}{c}
-I_{\mathrm{d}} \sin \delta-I_{\mathrm{q}} \cos \delta \\
I_{\mathrm{d}} \cos \delta-I_{\mathrm{q}} \sin \delta
\end{array}\right], \mathbf{T}_{\mathrm{V}}^{-1}=\left[\begin{array}{l}
-V_{\mathrm{D}} \sin \delta+V_{\mathrm{Q}} \cos \delta \\
-V_{\mathrm{D}} \cos \delta-V_{\mathrm{Q}} \sin \delta
\end{array}\right] .
\end{gathered}
$$

Thus, the complete model of the $\mathrm{SG}$ is derived:

$$
\begin{gathered}
\Delta \dot{\mathbf{X}}_{\mathrm{Gen}}=\mathbf{A}_{\mathrm{Gen}} \Delta \mathbf{X}_{\mathrm{Gen}}+\mathbf{B}_{\mathrm{Gen}} \Delta \mathbf{V}_{\mathrm{bDQ}}+\mathbf{B}_{\mathrm{Gen} \omega} \Delta \omega_{\mathrm{com}} \\
\Delta \mathbf{I}_{\mathrm{gDQ}}=\mathbf{C}_{\mathrm{Gen}} \Delta \mathbf{X}_{\mathrm{Gen}}, \Delta \omega=\mathbf{C}_{\mathrm{Gen} \omega} \Delta \mathbf{X}_{\mathrm{Gen}}
\end{gathered}
$$

where $\mathbf{X}_{\mathrm{Gen}}=\left[\omega, \delta_{\mathrm{Gen}}, \psi_{\mathrm{d}}, \psi_{\mathrm{q}}, \psi_{\mathrm{fd}}, \psi_{\mathrm{kq}}, \varepsilon_{\mathrm{v}}, T_{\mathrm{m}}, v_{\mathrm{fd}}\right]^{\mathrm{T}}, \mathbf{V}_{\mathrm{gDQ}}=\left[\nu_{\mathrm{D}}\right.$, $\left.v_{\mathrm{Q}}\right], \mathbf{I}_{\mathrm{gDQ}}=\left[i_{\mathrm{D}}, i_{\mathrm{Q}}\right], \mathbf{L}_{1}$ is the first two rows of matrix $\mathbf{L}, \mathbf{A}_{\mathrm{Gen}}=$ $\mathbf{A}_{\mathrm{g}}+\mathbf{B}_{\mathrm{g}} \mathbf{T}_{\mathrm{v}}^{-1}\left[0,1,0_{1 \times 7}\right], \mathbf{B}_{\mathrm{Gen} \omega}=\left[0,-\omega_{\mathrm{b}}, 0_{1 \times 7}\right]^{\mathrm{T}}, \mathbf{B}_{\mathrm{Gen}}=\mathbf{B}_{\mathrm{g}} \mathbf{T}^{-1}, \mathbf{C}_{\mathrm{Gen}}$ $=-\mathbf{T L}_{1}\left[0_{4 \times 2}, \mathrm{I}_{4 \times 4}, 0_{4 \times 3}\right]+\mathbf{T}_{\mathrm{c}}\left[0,1,0_{1 \times 7}\right], \mathbf{C}_{\mathrm{Gen} \omega}=\left[0,0_{1 \times 8}\right]$,

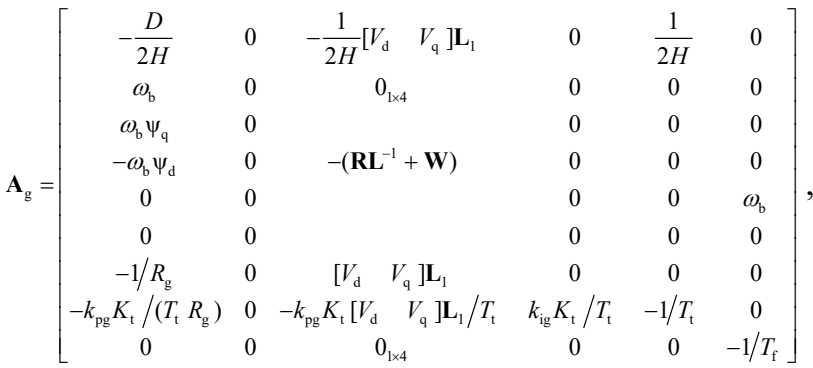

$$
\begin{aligned}
& \mathbf{B}_{\mathrm{g}}=\left[\begin{array}{ccccccc}
-\frac{I_{\mathrm{d}}}{2 H} & 0 & \omega_{\mathrm{b}} & 0 & -I_{\mathrm{d}} & -k_{\mathrm{pg}} K_{\mathrm{t}} I_{\mathrm{d}} / T_{\mathrm{t}} & -V_{\mathrm{gd}} K_{\mathrm{t}} V_{\mathrm{g}} / T_{\mathrm{t}} \\
-\frac{I_{\mathrm{q}}}{2 H} & 0 & 0 & \omega_{\mathrm{b}} & -I_{\mathrm{q}} & -k_{\mathrm{pg}} K_{\mathrm{f}} I_{\mathrm{q}} / T_{\mathrm{f}} & -V_{\mathrm{gq}} K_{\mathrm{t}} V_{\mathrm{g}} / T_{\mathrm{t}}
\end{array}\right]^{\mathrm{T}} .
\end{aligned}
$$

\section{B. Modeling the Grid-Following Inverter}

For GFL inverters, a multi-loop diagram, including the outer power loop control and the inner current loop control, is developed, as shown in Fig. 3 [1].

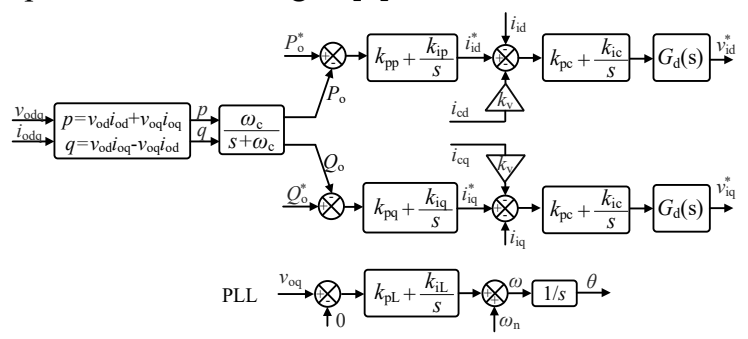

Fig. 3. Block diagram of GFL inverter controls.

Note that the active power, $P_{0}$, and reactive power, $Q_{0}$, in Fig. 3 are derived from the instantaneous values $p$ and $q$ using the low-pass filters (LPFs) with the cutoff frequency $\omega_{\mathrm{c}}$.

$$
P_{\mathrm{o}}=\frac{\omega_{\mathrm{c}}}{s+\omega_{\mathrm{c}}}\left(v_{\mathrm{od}} i_{\mathrm{od}}+v_{\mathrm{oq}} i_{\mathrm{oq}}\right), Q_{\mathrm{o}}=\frac{\omega_{\mathrm{c}}}{s+\omega_{\mathrm{c}}}\left(v_{\mathrm{od}} i_{\mathrm{oq}}-v_{\mathrm{oq}} i_{\mathrm{od}}\right)
$$

where $v_{\text {odq }}$ and $i_{\text {odq }}$ are inverter output voltages and currents, respectively. 
A phase angle difference $\delta_{\text {Inv }}$ is defined to represent the phase difference between the reference frame of the inverter and the common frame $D-Q$. Thus,

$$
\delta_{\text {Inv }}=\omega_{\mathrm{b}} \int\left(\omega_{\text {Inv }}-\omega_{\text {com }}\right) \mathrm{dt} \rightarrow \Delta \dot{\delta}_{\text {Inv }}=\omega_{\mathrm{b}} \Delta \omega_{\text {Inv }}-\omega_{\mathrm{b}} \Delta \omega_{\text {com }}
$$

For GFL inverters, the angular frequency and phase angle are determined by the external grids and they can be obtained through PLL, as shown in Fig. 3. By introducing an intermediate variable, $\varepsilon_{\mathrm{L}}$, it is obtained:

$$
\begin{gathered}
\dot{\varepsilon}_{\mathrm{L}}=v_{\mathrm{oq}}-0 \rightarrow \Delta \dot{\varepsilon}_{\mathrm{L}}=\Delta v_{\mathrm{oq}} \\
\omega_{\mathrm{Inv}}=k_{\mathrm{pL}}\left(v_{\mathrm{oq}}-0\right)+k_{\mathrm{iL}} \varepsilon_{\mathrm{L}}+\omega_{\mathrm{n}} \rightarrow \Delta \omega_{\mathrm{Inv}}=k_{\mathrm{pL}} \Delta v_{\mathrm{oq}}+k_{\mathrm{iL}} \Delta \varepsilon_{\mathrm{L}}
\end{gathered}
$$

Hence, the corresponding small-signal representations of $\varepsilon_{\mathrm{L}}$, $\delta_{\text {Inv }}, P_{\mathrm{o}}, Q_{\mathrm{o}}$, and $\omega_{\text {Inv }}$ are summarized as:

$$
\begin{gathered}
\Delta \dot{\mathbf{X}}_{\mathrm{P}}=\mathbf{A}_{\mathrm{P}} \mathbf{X}_{\mathrm{P}}+\mathbf{B}_{\mathrm{P}} \mathbf{X}_{\mathrm{FLL}}+\mathbf{B}_{\mathrm{P} \omega} \Delta \omega_{\text {com }} \\
\Delta \omega_{\text {Inv }}=\mathbf{C}_{\mathrm{P}_{\omega}} \mathbf{X}_{\mathrm{P}}+\mathbf{D}_{\mathrm{P}_{\omega}} \mathbf{X}_{\mathrm{FIL}}
\end{gathered}
$$

where $\mathbf{X}_{\mathrm{P}}=\left[\varepsilon_{\mathrm{L}}, \delta_{\mathrm{Inv}}, P_{\mathrm{o}}, Q_{\mathrm{o}}\right]^{\mathrm{T}}, \mathbf{X}_{\mathrm{FL}}=\left[i_{\mathrm{id}}, i_{\mathrm{iq}}, v_{\mathrm{od}}, v_{\mathrm{oq}}, i_{\mathrm{od}}, i_{\mathrm{oq}},\right]^{\mathrm{T}}$. For brevity, the detailed matrices (e.g., $\mathbf{A}_{\mathrm{p}}, \mathbf{B}_{\mathrm{p}}$ ) are not detailed here.

For the power control loop, the intermediate variables $\varphi_{\mathrm{d}}$ and $\varphi_{\mathrm{q}}$ are used to model the PI controllers:

$$
\begin{gathered}
\dot{\varphi}_{\mathrm{d}}=P_{\mathrm{o}}^{*}-P_{\mathrm{o}}, \dot{\varphi}_{\mathrm{q}}=Q_{\mathrm{o}}^{*}-Q_{\mathrm{o}} \\
i_{\mathrm{id}}^{*}=k_{\mathrm{pp}}\left(P_{\mathrm{o}}^{*}-P_{\mathrm{o}}\right)+k_{\mathrm{ip}} \varphi_{\mathrm{d}}, i_{\mathrm{iq}}^{*}=k_{\mathrm{pq}}\left(Q_{\mathrm{o}}^{*}-Q_{\mathrm{o}}\right)+k_{\mathrm{iq}} \varphi_{\mathrm{q}}
\end{gathered}
$$

Note that when the frequency and voltage droop control are used to support GFL control, the references of the active and reactive power can be generated as follows:

$$
P_{\mathrm{o}}^{*}=P_{\mathrm{n}}-\left(\omega_{\text {Inv }}-\omega_{\mathrm{n}}\right) / m_{\mathrm{p}}, Q_{\mathrm{o}}^{*}=Q_{\mathrm{n}}-\left(V_{\text {olnv }}-V_{\mathrm{n}}\right) / n_{\mathrm{q}}
$$

where $P_{\mathrm{n}}, Q_{\mathrm{n}}$ are the set points; $m_{\mathrm{p}}, n_{\mathrm{p}}$ are the droop coefficients.

When voltage magnitude is oriented to the $d$-axis, it yields:

$$
v_{\text {od }}=V_{\text {olnv }}
$$

Thus, the corresponding small-signal representation of the outer power loop is derived as:

$$
\begin{array}{r}
\Delta \dot{\mathbf{X}}_{\varphi}=0_{2 \times 2} \Delta \mathbf{X}_{\varphi}+\mathbf{B}_{\mathrm{S} 1} \Delta \mathbf{X}_{\mathrm{P}}+\mathbf{B}_{\mathrm{S} 2} \Delta \mathbf{X}_{\mathrm{FLL}}+\mathbf{B}_{\mathrm{S} \omega} \Delta \omega_{\mathrm{Inv}} \\
\Delta \mathbf{I}_{\mathrm{idq}}^{*}=\mathbf{C}_{\mathrm{S}} \Delta \mathbf{X}_{\varphi}+\mathbf{D}_{\mathrm{S} 1} \Delta \mathbf{X}_{\mathrm{P}}+\mathbf{D}_{\mathrm{S} 2} \Delta \mathbf{X}_{\mathrm{FLL}}+\mathbf{D}_{\mathrm{S \omega} \omega} \Delta \omega_{\mathrm{Inv}}
\end{array}
$$

where $\mathbf{X}_{\varphi}=\left[\varphi_{\mathrm{d}}, \varphi_{\mathrm{q}}\right]^{\mathrm{T}}, \mathbf{I}_{\mathrm{idq}}^{*}=\left[i_{\mathrm{id}}^{*}, i_{\mathrm{iq}}^{*}\right]^{\mathrm{T}}$.

If the active and reactive power references are constant, $\mathbf{B}_{\mathrm{s} 2}$ $=0_{2 \times 6}, \mathbf{B}_{\mathrm{s} \omega}=0_{2 \times 1}, \mathbf{D}_{\mathrm{s} 2}=0_{2 \times 6}, \mathbf{D}_{\mathrm{s} \omega}=0_{2 \times 1}$.

For the current control loop, the intermediate variables $\tau_{\mathrm{d}}$ and $\tau_{\mathrm{q}}$ are used to model the PI controllers. Further, the capacitorcurrent-feedback-based active damping is used to address the resonance issue introduced by the LCL filter [10]. Thus,

$$
\begin{gathered}
\dot{\tau}_{\mathrm{dq}}=i_{\mathrm{idq}}^{*}-i_{\mathrm{idq}} \\
v_{\mathrm{idq}}^{*}=G_{\mathrm{d}}(s)\left[k_{\mathrm{pc}}\left(i_{\mathrm{idq}}^{*}-i_{\mathrm{idq}}\right)+k_{\mathrm{ic}} \tau_{\mathrm{dq}}-k_{\mathrm{v}}\left(i_{\mathrm{idq}}-i_{\mathrm{odq}}\right)\right]
\end{gathered}
$$

where $k_{v}$ is the capacitor-current-feedback gain. Note that a zeroorder holding delay is used to represent the pulse width modulator in (27), which is approximated as follows:

$$
G_{\mathrm{d}}(s)=e^{-1.5 T_{\mathrm{s}} s} \approx \frac{1}{1+1.5 T_{\mathrm{s}} s}=G_{\mathrm{LPF}}(s)=\frac{\omega_{\mathrm{t}}}{s+\omega_{\mathrm{t}}}
$$

where $T_{\mathrm{s}}$ is the sampling time, $\omega_{\mathrm{t}}$ is the equivalent cut-off frequency, which equals $1 /\left(1.5 T_{\mathrm{S}}\right)$. Thus, the small-signal model for the inner current loop together with the pulse width modulator is derived:

$$
\Delta \dot{\mathbf{X}}_{\tau}=\mathbf{0}_{2 \times 2} \Delta \mathbf{X}_{\tau}+\mathbf{B}_{\mathrm{C} 1} \Delta \mathbf{I}_{\mathrm{idq}}^{*}+\mathbf{B}_{\mathrm{C} 2} \Delta \mathbf{X}_{\mathrm{FIL}}
$$

$$
\Delta \dot{\mathbf{V}}_{\mathrm{idq}}^{*}=\mathbf{A}_{\mathrm{T}} \Delta \mathbf{V}_{\mathrm{idq}}^{*}+\mathbf{B}_{\mathrm{T} 1} \Delta \mathbf{I}_{\mathrm{fdq}}^{*}+\mathbf{B}_{\mathrm{T} 2} \mathbf{X}_{\tau}+\mathbf{B}_{\mathrm{T} 3} \Delta \mathbf{X}_{\mathrm{FLL}}
$$

where $\mathbf{X}_{\tau}=\left[\tau_{\mathrm{d}}, \tau_{\mathrm{q}}\right]^{\mathrm{T}}, \mathbf{V}_{\mathrm{idq}}^{*}=\left[v_{\mathrm{id}}^{*}, v_{\mathrm{id}}^{*}\right]^{\mathrm{T}}$.

The filter is modeled as follows by assuming that the inverter generates the required voltage $\left(v_{\mathrm{i}}=v_{\mathrm{i}}^{*}\right)$ :

$$
\begin{gathered}
L_{\mathrm{i}} \dot{i}_{\mathrm{ddq}}=\omega_{\mathrm{b}}\left(v_{\mathrm{idq}}-v_{\mathrm{odq}}-R_{\mathrm{i}} i_{\mathrm{idq}} \pm \omega_{\mathrm{Inv}} L_{\mathrm{i}} i_{\mathrm{iqd}}\right) \\
C_{\mathrm{f}} \dot{v}_{\mathrm{odq}}=\omega_{\mathrm{b}}\left(i_{\mathrm{idq}}-i_{\mathrm{odq}} \pm \omega_{\mathrm{Inv}} C_{\mathrm{f}} v_{\mathrm{oqd}}\right) \\
L_{\mathrm{c}} i_{\mathrm{odq}}=\omega_{\mathrm{b}}\left(v_{\mathrm{odq}}-v_{\mathrm{cdq}}-R_{\mathrm{c}} i_{\text {odq }} \pm \omega_{\mathrm{Inv}} L_{\mathrm{c}} i_{\text {oqd }}\right)
\end{gathered}
$$

The small-signal representation is shown:

$$
\dot{\mathbf{X}}_{\mathrm{FLL}}=\mathbf{A}_{\mathrm{FLL}} \mathbf{X}_{\mathrm{FIL}}+\mathbf{B}_{\mathrm{FLL} 1} \mathbf{V}_{\mathrm{idq}}+\mathbf{B}_{\mathrm{FIL} 2} \mathbf{V}_{\mathrm{cdq}}+\mathbf{B}_{\mathrm{FL} \omega} \Delta \omega_{\mathrm{Inv}}
$$

Thus, the complete small-signal model of the grid-following inverter can be expressed as:

$$
\begin{gathered}
\Delta \dot{\mathbf{X}}_{\text {Inv }}=\mathbf{A}_{\text {Inv }} \Delta \mathbf{X}_{\text {Inv }}+\mathbf{B}_{\text {Inv }} \Delta \mathbf{V}_{\mathrm{cDQ}}+\mathbf{B}_{\text {Inv }} \Delta \omega_{\text {com }} \\
\Delta \mathbf{I}_{\text {oDQ }}=\mathbf{C}_{\text {Inv }} \Delta \mathbf{X}_{\text {Inv }}
\end{gathered}
$$

where $\mathbf{X}_{\mathrm{Inv}}=\left[\mathbf{X}_{\mathrm{P}}, \mathbf{X}_{\varphi}, \mathbf{X}_{\tau}, \mathbf{V}_{\mathrm{i}}, \mathbf{X}_{\mathrm{FIL}}\right]^{\mathrm{T}}, \mathbf{V}_{\mathrm{cDQ}}=\left[v_{\mathrm{cD}}, v_{\mathrm{cQ}}\right], \mathbf{I}_{\mathrm{oDQ}}=\left[i_{\mathrm{oD}}\right.$, $i_{\mathrm{oQ}}$,

$$
\mathbf{A}_{\mathrm{Inv}}=\left[\begin{array}{ccccc}
\mathbf{A}_{\mathrm{P}} & 0_{4 \times 2} & 0_{4 \times 2} & 0_{4 \times 2} & \mathbf{B}_{\mathrm{P}} \\
\mathbf{B}_{\mathrm{S} 1}+\mathbf{B}_{\mathrm{S} \omega} \mathbf{C}_{\mathrm{P} \omega} & 0_{2 \times 2} & 0_{2 \times 2} & 0_{2 \times 2} & \mathbf{B}_{\mathrm{S} 2}+\mathbf{B}_{\mathrm{S} \omega} \mathbf{D}_{\mathrm{P} \omega} \\
\mathbf{B}_{\mathrm{C} 1}\left(\mathbf{D}_{\mathrm{S} 1}+\mathbf{D}_{\mathrm{S} \omega} \mathbf{C}_{\mathrm{P} \omega}\right) & \mathbf{B}_{\mathrm{C} 1} \mathbf{C}_{\mathrm{S}} & 0_{2 \times 2} & 0_{2 \times 2} & \mathbf{B}_{\mathrm{C} 2}+\mathbf{B}_{\mathrm{C} 1}\left(\mathbf{D}_{\mathrm{S} 2}+\mathbf{D}_{\mathrm{S} \omega} \mathbf{D}_{\mathrm{P} \omega}\right. \\
\mathbf{B}_{\mathrm{T} 1}\left(\mathbf{D}_{\mathrm{S} 1}+\mathbf{D}_{\mathrm{S} \omega} \mathbf{C}_{\mathrm{P} \omega}\right) & \mathbf{B}_{\mathrm{T} 1} \mathbf{C}_{\mathrm{S}} & \mathbf{B}_{\mathrm{T} 2} & \mathbf{A}_{\mathrm{T}} & \mathbf{B}_{\mathrm{T} 3}+\mathbf{B}_{\mathrm{T} 1}\left(\mathbf{D}_{\mathrm{S} 2}+\mathbf{D}_{\mathrm{S} \omega} \mathbf{D}_{\mathrm{P} \omega}\right) \\
\mathbf{B}_{\mathrm{FIL} 2} \mathbf{T}_{\mathrm{V}}^{-1}\left[\begin{array}{lll}
0 & 1 & 0
\end{array}\right]+\mathbf{B}_{\mathrm{FIL} \omega} \mathbf{C}_{\mathrm{P} \omega} & 0_{6 \times 2} & 0_{6 \times 2} & \mathbf{B}_{\mathrm{FL} 1} & \mathbf{A}_{\mathrm{FIL}}+\mathbf{B}_{\mathrm{FIL} \omega} \mathbf{D}_{\mathrm{P} \omega}
\end{array}\right]
$$

$\mathbf{B}_{\text {Inv }}=\left[0_{4 \times 2}, 0_{2 \times 2}, 0_{2 \times 2}, 0_{2 \times 2}, \mathbf{B}_{\text {Inv }} \mathbf{T}^{-1}\right]^{\mathrm{T}}, \mathbf{B}_{\text {Inv } \omega}=\left[\mathbf{B}_{\text {P } \omega}, 0_{2 \times 1}, 0_{2 \times 1}, 0_{2 \times 1}\right.$, $\left.0_{6 \times 1}\right]^{\mathrm{T}}, \mathbf{C}_{\text {Inv }}=\left[\mathbf{T}_{C}[0,1,0,0], 0_{2 \times 2}, 0_{2 \times 2}, 0_{2 \times 2}, 0_{2 \times 2}, 0_{2 \times 2}, \mathbf{T}\right]$.

\section{Modeling the Grid-Forming Inverter}

Similar to GFL inverter, a multi-loop diagram-including the droop control, the outer voltage loop, and the inner current loop-is developed for the GFM inverter, as shown in Fig. 4 [1].

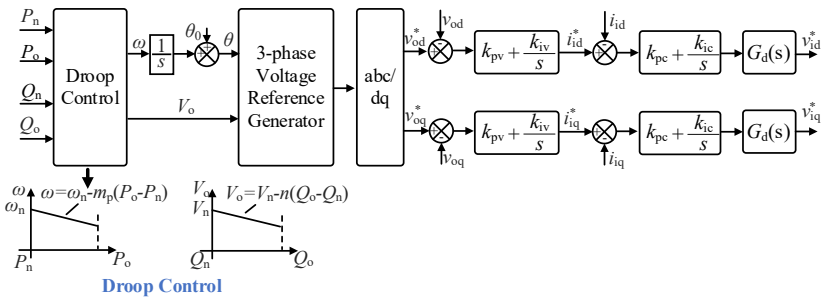

Fig. 4. Block diagram of GFM inverter controls.

For the GFM inverter, when the voltage magnitude is oriented to the $d$-axis, it yields:

$$
\begin{gathered}
\omega_{\text {Inv }}=\omega_{\mathrm{n}}-m_{\mathrm{p}}\left(P_{\mathrm{o}}-P_{\mathrm{n}}\right) \rightarrow \Delta \omega_{\mathrm{Inv}}=-m_{\mathrm{p}} \Delta P_{\mathrm{o}} \\
v_{\mathrm{od}}^{*}=V_{\mathrm{n}}-n_{\mathrm{q}}\left(Q_{\mathrm{o}}-Q_{\mathrm{n}}\right), \quad v_{\mathrm{oq}}^{*}=0 \rightarrow \Delta v_{\mathrm{od}}^{*}=-n_{\mathrm{q}} \Delta Q_{\mathrm{o}}, \Delta v_{\mathrm{oq}}^{*}=0
\end{gathered}
$$

Thus, the corresponding small-signal representations of $\delta_{\text {Inv }}$, $P_{\mathrm{o}}, Q_{\mathrm{o}}$ are summarized as:

$$
\begin{aligned}
& \Delta \dot{\mathbf{X}}_{\mathrm{P}}^{\prime}=\mathbf{A}_{\mathrm{P}}^{\prime} \mathbf{X}_{\mathrm{P}}^{\prime}+\mathbf{B}_{\mathrm{P}}^{\prime} \mathbf{X}_{\mathrm{FIL}}+\mathbf{B}_{\mathrm{P} \omega}^{\prime} \Delta \omega_{\text {com }} \\
& \Delta \mathbf{V}_{\text {odq }}^{*}=\mathbf{C}_{\mathrm{P}}^{\prime} \Delta \mathbf{X}_{\mathrm{P}}^{\prime}, \Delta \omega_{\text {Inv }}=\mathbf{C}_{\mathrm{P} \omega}^{\prime} \Delta \mathbf{X}_{\mathrm{P}}
\end{aligned}
$$

where $\mathbf{X}_{\mathrm{P}}^{\prime}=\left[\delta_{\text {Inv }}, P_{\mathrm{o}}, Q_{\mathrm{o}}\right]^{\mathrm{T}}, \mathbf{V}_{\mathrm{odq}}^{*}=\left[v_{\mathrm{od}}^{*}, v_{\mathrm{oq}}^{*}\right]^{\mathrm{T}}$.

For the voltage control loop, the intermediate variables $\varphi_{\mathrm{d}}$ and $\varphi_{\mathrm{q}}$ are used to model the PI controllers in the $d$ - and $q$-axis:

$$
\begin{gathered}
\dot{\varphi}_{\mathrm{d}}=v_{\mathrm{od}}^{*}-v_{\mathrm{od}}, \dot{\varphi}_{\mathrm{q}}=v_{\mathrm{oq}}^{*}-v_{\mathrm{oq}} \\
i_{\mathrm{id}}^{*}=k_{\mathrm{pv}}\left(v_{\mathrm{od}}^{*}-v_{\mathrm{od}}\right)+k_{\mathrm{iv}} \varphi_{\mathrm{d}}, i_{\mathrm{iq}}^{*}=k_{\mathrm{pv}}\left(v_{\mathrm{oq}}^{*}-v_{\mathrm{oq}}\right)+k_{\mathrm{iv}} \varphi_{\mathrm{q}}
\end{gathered}
$$

Thus, the small-signal representation of the voltage loop is:

$$
\Delta \dot{\mathbf{X}}_{\varphi}=0_{2 \times 2} \Delta \mathbf{X}_{\varphi}+\mathbf{B}_{\mathrm{V} 1} \Delta \mathbf{V}_{\text {odq }}^{*}+\mathbf{B}_{\mathrm{V} 2} \Delta \mathbf{X}_{\mathrm{FIL}}
$$




$$
\Delta \mathbf{I}_{\mathrm{idq}}^{*}=\mathbf{C}_{\mathrm{V} 1} \Delta \mathbf{X}_{\varphi}+\mathbf{D}_{\mathrm{V} 1} \Delta \mathbf{V}_{\text {odq }}^{*}+\mathbf{D}_{\mathrm{V} 2} \Delta \mathbf{X}_{\mathrm{FL}}
$$

For the other parts, the small-signal modeling of the GFM inverter is the same as that of the GFL, except that the active damping loop is disabled by seting $k_{\mathrm{v}}=0$; hence, they are neglected for simplification.

Finally, the complete small-signal model of the GFM inverter can be expressed as:

$$
\begin{gathered}
\Delta \dot{\mathbf{X}}_{\text {Inv }}=\mathbf{A}_{\text {Inv }} \Delta \mathbf{X}_{\text {Inv }}+\mathbf{B}_{\text {Inv }} \Delta \mathbf{V}_{\mathrm{cDQ}}+\mathbf{B}_{\text {Inv }} \Delta \omega_{\mathrm{com}} \\
\Delta \mathbf{I}_{\mathrm{oDQ}}=\mathbf{C}_{\mathrm{Inv}} \Delta \mathbf{X}_{\mathrm{Inv}}
\end{gathered}
$$

where $\mathbf{X}_{\mathrm{Inv}}=\left[\mathbf{X}_{\mathrm{p}}^{\prime}, \mathbf{X}_{\varphi}, \mathbf{X}_{\tau}, \mathbf{V}_{\mathrm{i}}, \mathbf{X}_{\mathrm{FIL}}\right]^{\mathrm{T}}$,

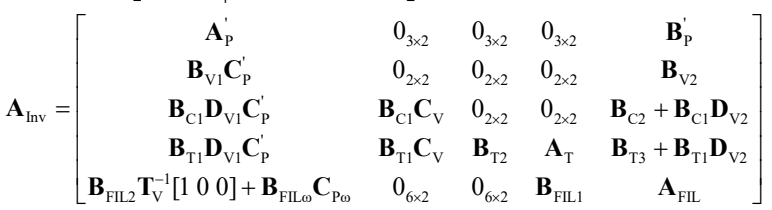

$\mathbf{B}_{\text {Inv }}=\left[0_{3 \times 2}, 0_{2 \times 2}, 0_{2 \times 2}, 0_{2 \times 2}, \mathbf{B}_{\text {Inv }} \mathbf{T}^{-1}\right]^{\mathrm{T}}, \mathbf{B}_{\text {Inv }}=\left[\mathbf{B}_{\mathrm{P}_{\omega}}^{\prime}, 0_{2 \times 1}, 0_{2 \times 1}, 0_{2 \times 1}\right.$, $\left.0_{6 \times 1}\right]^{\mathrm{T}}, \mathbf{C}_{\mathrm{Inv}}=\left[\mathbf{T}_{\mathrm{C}}[1,0,0], 0_{2 \times 2}, 0_{2 \times 2}, 0_{2 \times 2}, 0_{2 \times 2}, 0_{2 \times 2}, \mathbf{T}\right]$.

\section{Modeling the Network and Loads}

The network interconnects the IBR and SG, as well as the loads. The constant RL impedances are used to model the networks and the load center, and the constant impedance loads are used for local loads on the SG and inverter sides. Thus:

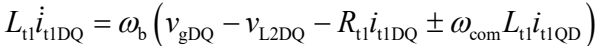

$$
\begin{aligned}
& L_{\mathrm{t} 2} i_{\mathrm{t} 2 \mathrm{DQ}}=\omega_{\mathrm{b}}\left(v_{\mathrm{cDQ}}-v_{\mathrm{L} 2 \mathrm{DQ}}-R_{\mathrm{t} 2} i_{\mathrm{t} \mathrm{DQ}} \pm \omega_{\mathrm{com}} L_{\mathrm{t} 2} i_{\mathrm{t} 2 \mathrm{DD}}\right) \\
& L_{\mathrm{L} 2} i_{\mathrm{L} 2 \mathrm{DQ}}=\omega_{\mathrm{b}}\left(v_{\mathrm{L} 2 \mathrm{DQ}}-R_{\mathrm{L} 2} i_{\mathrm{L} 2 \mathrm{DQ}} \pm \omega_{\mathrm{com}} L_{\mathrm{L} 2} i_{\mathrm{L} 2 \mathrm{QD}}\right)
\end{aligned}
$$

where $v_{\mathrm{L} 2 \mathrm{DQ}}$ are the bus voltages of the load center.

The small-signal model is thereby summarized as:

$$
\begin{gathered}
\Delta \dot{\mathbf{I}}_{\mathrm{NetDQ}}=\mathbf{A}_{\text {Net }} \Delta \mathbf{I}_{\text {NetDQ }}+\mathbf{B}_{\text {Net }} \Delta \mathbf{V}_{\text {bDQ }}+\mathbf{B}_{\text {Neto }} \Delta \omega_{\text {com }} \\
\Delta \dot{\mathbf{I}}_{\text {LoadDQ }}=\mathbf{A}_{\text {Load }} \Delta \mathbf{I}_{\text {LoadDQ }}+\mathbf{B}_{\text {Load }} \Delta \mathbf{V}_{\text {bDQ }}+\mathbf{B}_{\text {Load }} \Delta \omega_{\text {com }}
\end{gathered}
$$

where $\mathbf{I}_{\mathrm{NetDQ}}=\left[i_{11 \mathrm{D}}, i_{11 \mathrm{Q}}, i_{2 \mathrm{D}}, i_{\mathrm{t} 2 \mathrm{Q}}\right]^{\mathrm{T}}, \mathbf{I}_{\mathrm{LoadDQ}}=\left[i_{\mathrm{L} 2 \mathrm{D}}, i_{\mathrm{L} 2 \mathrm{Q}}\right]^{\mathrm{T}}, \mathbf{V}_{\mathrm{bDQ}}=$ $\left[v_{\mathrm{gD}}, v_{\mathrm{gQ}}, v_{\mathrm{L} 2 \mathrm{D}}, v_{\mathrm{L} 2 \mathrm{Q}}, v_{\mathrm{cD}}, v_{\mathrm{cQ}}\right]^{\mathrm{T}}$.

\section{E. Complete System Model}

To derive the complete system model, a large virtual resistance, $R_{\mathrm{N}}$, is involved to represent the resistance between the load center and the ground [11]; therefore:

$$
\begin{gathered}
\Delta v_{\mathrm{gDQ}}=R_{\mathrm{L} 1}\left(\Delta i_{\mathrm{gDQ}}-\Delta i_{\mathrm{t} 1 \mathrm{QQ}}\right) \\
\Delta v_{\mathrm{L} 2 \mathrm{DQ}}=R_{\mathrm{N}}\left(\Delta i_{\mathrm{L} 2 \mathrm{DQ}}-\Delta i_{\mathrm{t} 1 \mathrm{QQ}}-\Delta i_{\mathrm{t} 2 \mathrm{DQ}}\right) \\
\Delta v_{\mathrm{cDQ}}=R_{\mathrm{L} 3}\left(\Delta i_{\mathrm{oDQ}}-\Delta i_{\mathrm{t} \mathrm{DQ}}\right)
\end{gathered}
$$

Hence, the small-signal representation is derived as:

$$
\Delta \mathbf{V}_{\mathrm{bDQ}}=\mathbf{M}_{\mathrm{inv}} \Delta \mathbf{I}_{\mathrm{oDQ}}+\mathbf{M}_{\mathrm{Net}} \Delta \mathbf{I}_{\mathrm{NetDQ}}+\mathbf{M}_{\mathrm{Load}} \Delta \mathbf{I}_{\text {LoadDQ }}
$$

Therefore, by combing the modeling of the SG, IBR, loads, and networks, the entire system model is derived as:

$$
\dot{\mathbf{X}}_{\mathrm{sys}}=\mathbf{A}_{\text {sys }} \mathbf{X}_{\text {sys }}
$$

where $\mathbf{X}_{\text {sys }}=\left[\Delta \mathbf{X}_{\mathrm{Gen}}, \Delta \mathbf{X}_{\text {Inv }}, \Delta \mathbf{X}_{\mathrm{Load}}, \Delta \mathbf{X}_{\mathrm{Net}}\right]$, and

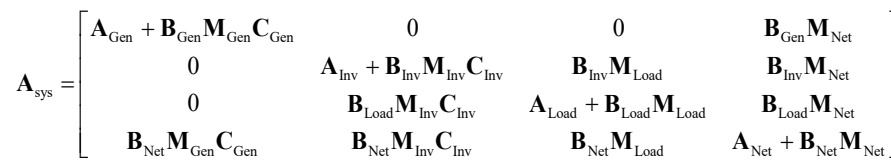

Further, the small-signal modeling analysis is based on the given operating points, which are obtained through power flow calculation. Note that the revised power flow calculation in [12] is used because GFM inverters are included.

\section{MODAL ANALYSIS OF GRID-FOLLOWING AND GRID- FORMING INVERTERS}

The eigenvalues of the entire system can be obtained by using (55). For a quantitative study, the IBR penetration level is defined as the ratio of the active power provided by IBRs and the total load active power at the rated condition:

$$
\eta=\frac{P_{\text {olnv }}}{P_{\text {nLoad }}} \times 100 \%
$$

The participation factors are used to identify the impacts of state variables on the corresponding eigenvalues [2]. A comparative study is conducted to investigate the impacts of different kinds of inverter control algorithms (i.e., GFL without droop, GFL with droop, and GFM control) on power system small-signal stability. For the modal analysis in this section, the IBR penetration levels in these three cases are set to be identical at $75 \%$. The SG-side and IBR-side transmission line lengths are set at $50 \mathrm{~km}$ and $1 \mathrm{~km}$, respectively. The voltage level of the transmission line is chosen as $230 \mathrm{kV}$.

TABLE I. CRITICAL OSCILLATORY MODES OF GFL INVERTER (WITHOUT DROOP) AND SG

\begin{tabular}{|c|c|c|c|c|c|}
\hline Eigenvalues & Real & Imag. & Freq. & Damping Ratio & Type \\
\hline 1, 2 (Mode 1) & -106.83 & 496.49 & 79.02 & 0.21 & Coupling \\
\hline 3, 4 (Mode 2) & -132.60 & 146.63 & 23.34 & 0.67 & Inverter local \\
\hline 5, 6 (Mode 3) & -15.39 & 128.09 & 20.39 & 0.12 & Weak coupling \\
\hline 7,8 (Mode 4) & -2.00 & 23.07 & 3.67 & 0.09 & SG local \\
\hline 9, 10 (Mode 5) & -0.69 & 1.57 & 0.25 & 0.40 & SG local \\
\hline
\end{tabular}

TABLE II. CRITICAL OSCILLATORY MODES OF GFL INVERTER (WITH DROOP)

\begin{tabular}{|c|c|c|c|c|c|}
\hline Eigenvalues & Real & Imag. & Freq. & Damping Ratio & Type \\
\hline 1, 2 (Mode 1) & -108.81 & 493.26 & 78.50 & 0.22 & Coupling \\
\hline 3, 4 (Mode 2) & -126.99 & 143.80 & 22.89 & 0.66 & Inverter local \\
\hline 5,6 (Mode 3) & -41.59 & 115.82 & 18.43 & 0.34 & Weak coupling \\
\hline 7,8 (Mode 4) & -2.14 & 22.91 & 3.65 & 0.09 & SG local \\
\hline 9,10 (Mode 5) & -0.58 & 1.75 & 0.28 & 0.32 & Weak coupling \\
\hline
\end{tabular}

TABLE III. CRITICAL OSCILLATORY MODES OF GFM INVERTER AND SG

\begin{tabular}{|c|c|c|c|c|c|}
\hline Eigenvalues & Real & Imag. & Freq. & Damping Ratio & Type \\
\hline 1,2 (Mode 1) & -125.70 & 442.05 & 70.35 & 0.27 & Coupling \\
\hline 3,4 (Mode 3) & -35.86 & 74.47 & 11.85 & 0.43 & Inverter local \\
\hline 5,6 (Mode 4) & -1.27 & 23.00 & 3.66 & 0.05 & SG local \\
\hline 7,8 (Mode 5) & -0.52 & 1.05 & 0.16 & 0.45 & Weak coupling \\
\hline 9,10 (Mode 6) & -1.63 & 5.87 & 0.93 & 0.27 & Coupling \\
\hline
\end{tabular}

For the comparative analysis, only oscillatory modes with small real part or low damping ratio are chosen. Based on the results shown in Table I-Table III and Fig. 5 - Fig. 7, it is found that the responses of Mode 1, Mode 3, and Mode 4 of the selected three types of control algorithms are similar. Mode 1 is mainly impacted by the flux linkage of the SG, IBR side current, and network currents, and thus it is identified as the coupling mode. Mode 3 is mainly affected by the IBR inner current loop for all three types of IBR controls, whereas this mode is weakly affected by the flux linkage of the SG for the GFL inverter, and thus it is identified as the weak coupling mode. Mode 4 is mainly influenced by the flux linkage and voltage of the exciter, and thus it is identified as the local SG mode. Additionally, because of the implementation of the PLL in the GFL inverters, there is one mode (i.e., Mode 2) that exist only in GFL inverters. Further, the coupling of the SG and IBR can be founded in Mode 5 for both the control algorithm GFL with droop and GFM. It is observed that the outer voltage loop of the GFM inverter is weakly coupled with the governor and turbine of the SG whereas the outer power loop of the GFL inverter with droop is weakly coupled with the SG governor and turbine. Finally, it is found that there is another unique coupling mode (i.e., Mode 6) in the GFM inverters; this is mainly impacted by the SG output frequency and the IBR output phase angle. 

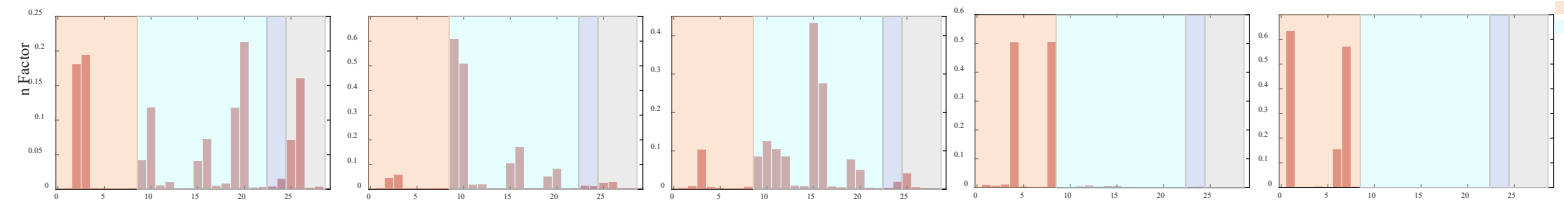

Fig. 5. Participation factor analysis for GFL inverter without droop. (a) Mode 1. (b) Mode 2. (c) Mode 3. (d) Mode 4. (e) Mode 5.
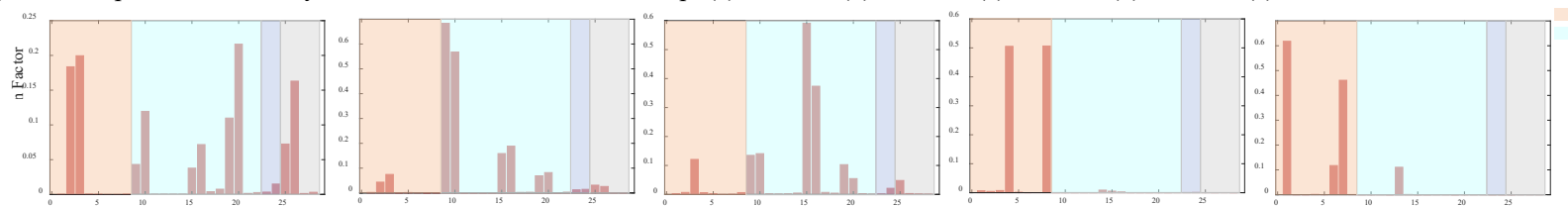

Fig. 6. Participation factor analysis for GFL inverter with droop. (a) Mode 1. (b) Mode 2. (c) Mode 3. (d) Mode 4. (e) Mode 5.
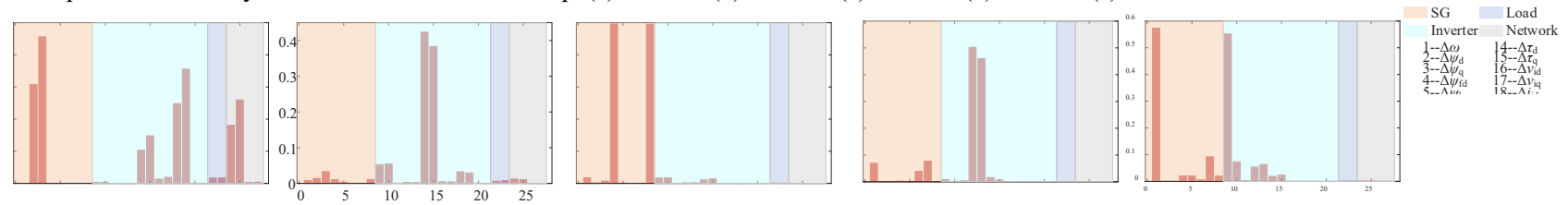

Fig. 7. Participation factor analysis for GFM inverter. (a) Mode 1. (b) Mode 3. (c) Mode 4. (d) Mode 5. (e) Mode 6.

IV. Sensitivity ANAlysis AND CASE STUDIES

This section investigates the impact of the length of the transmission line, the IBR penetration level, and other key control parameters on small-signal stability are investigated for GFL inverter without droop and GFM inverter, the GFL inverter with droop are similar to that of the GFL inverter without droop; hence it will be not included in this section for brevity.

\section{A. Impact of Length of the Transmission Line}

The length of the SG side transmission line was varied from $100 \mathrm{~km}$ to $400 \mathrm{~km}$. As shown in Fig. 8, a shorter transmission line can support a higher penetration level of the inverter in terms of small-signal stability for both GFL and GFM inverters.
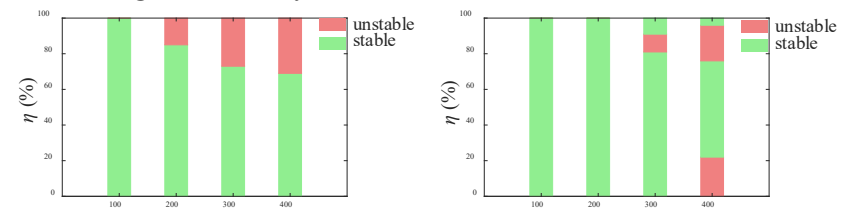

Fig. 8. Impact of the length of the transmission line on the penetration level of the (a) GFL inverter and (b) GFM inverter.

TABLE IV. SCR AT LOAD BUS WITH VARYING LENGTH OF SG SIDE TRANSMISSION LINE

\begin{tabular}{|c|c|}
\hline Line Length $(\mathrm{km})$ & SCR \\
\hline 100 & 2.19 \\
\hline 200 & 1.44 \\
\hline 300 & 1.07 \\
\hline 400 & 0.85 \\
\hline
\end{tabular}

Further, it is observed that there is an upper boundary in the penetration level of the GFL inverter, whereas there is a gap and a low boundary on the penetration level of the GFM inverter, both under long transmission lines, indicating how IBRs affect small-signal stability under different control algorithms. It is also shown that it could be easier to achieve $100 \%$ penetration level with small-signal stability guarantees for the GFM inverter, though there could be additional operating constraints for relatively low penetration levels.

Note that a short-circuit ratio (SCR) can be used to assess the strength or voltage stiffness of a particular point of interconnection. A low SCR indicates a low grid strength condition [13]. The SCRs for varying transmission line lengths are provided in Table IV.

From Table IV and Fig. 8, it is shown that a stronger point can support a higher IBR penetration level in terms of smallsignal stability for both GFL and GFM inverters. A low SCR could require additional design considerations.

\section{B. Impact of Penetration Level}

Eigenvalue analysis for identifying the impacts of IBR penetration level on small-signal stability is conducted for both GFL (without droop) and GFM inverters under the SCR of 0.85, as shown in Fig. 9 and Fig. 10. Note that the increase in IBR penetration level usually accompanies the displacement of the SG, and thus the system inertia decreases accordingly; thus, the inertia constant is set to be proportional to the SG output active power:

$$
H=P_{\text {oGen }} H_{0}
$$

The IBR penetration level was varied from 0.05 to 0.95 . Results show that Mode 3 oscillation has the dominant impacts on small-signal stability for GFL inverters when the penetration level changes, whereas Mode 6 has the dominant impact on small-signal stability for GFM inverters.

Time-domain EMT tests around the stability boundary were also provided to verify the this analysis, as shown in Fig. 11 and Fig. 12. To test the small-signal stability, a 5\% drop in the load was applied at $t=5 \mathrm{~s}$ for all stable cases. Results show that the time-domain tests are consistent with the theoretical analysis.

\section{Impact of Control Parameters}

Fig. 13 shows the damping ratios of Mode 3 for GFL inverters and Mode 6 for GFM inverters with varying control parameters, where less-than-zero damping ratio indicates an unstable system. By tuning the integral gain $k_{\text {ic }}$ of the GFL inverter current loop from 50 to 30 , the less-than-zero damping ratio can be gradually shifted to a positive value, which means that the system can support a higher penetration level of IBRs with small-signal stability guarantees. Further, the 100\% penetration level of the GFM inverter can be achieved by tuning 
the integral gain $k_{\text {iv }}$ of the inverter voltage loop from 2.0 to 0.5 and the gaps that exist in Fig. 8 (b) can be thereby eliminated.

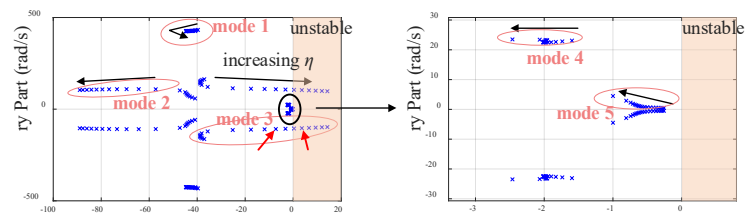

Fig. 9. (a) Eigenvalues trajectories for different penetration levels of GFL inverters without droop. (b) Zoom-in results.

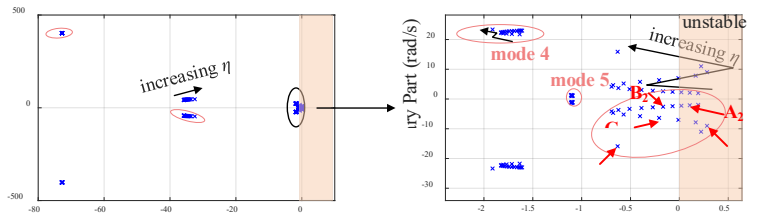

Fig. 10. (A) Eigenvalues trajectories for different penetration levels of GFM inverters. (b) Zoom-in results.

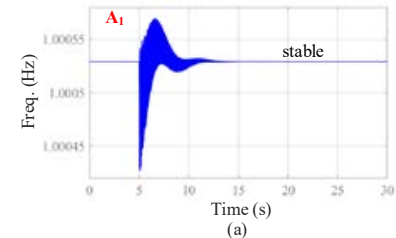

(a)

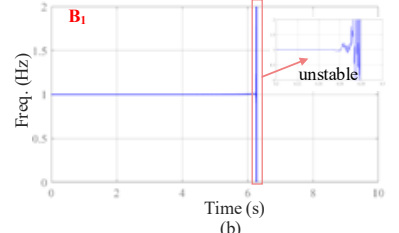

(b)
Fig. 11. Time-domain tests of GFL inverters. (a) Point $A_{1}$ (b) Point $B_{1}$

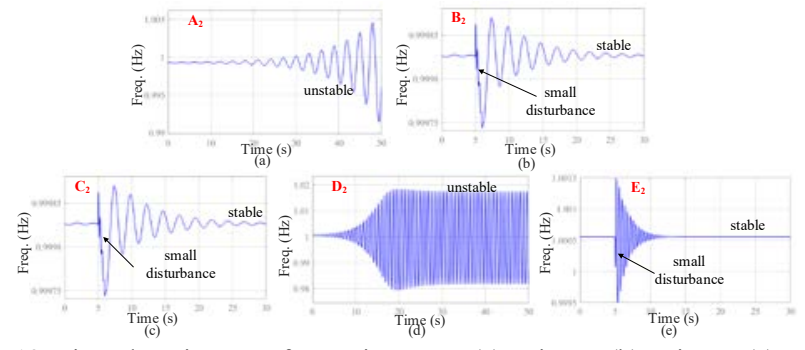

Fig. 12. Time-domain tests of GFM inverters. (a) Point $\mathrm{A}_{2}$ (b) Point $\mathrm{B}_{2}$ (c) Point $\mathrm{C}_{2}$ (d) Point $\mathrm{D}_{2}$ (e) Point $\mathrm{E}_{2}$.

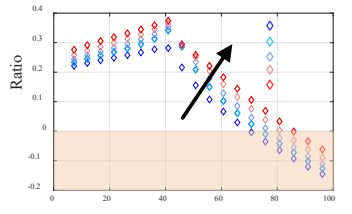

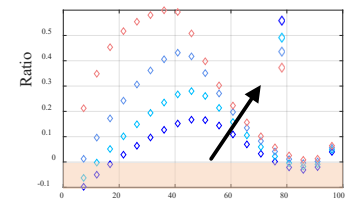

Fig. 13. Damping ratio with varying control parameters of (a) GFL inverters and (b) GFM inverters.

\section{CONCLUSION}

This paper conducted small-signal stability analysis of a simplified power grid with GFL and GFM IBRs and SGs. The investigation reveals the different coupling mechanisms between IBRs and SGs on small-signal stability under GFM and GFL control scenarios. The paper also evaluated the impact of key parameters on system stability, including the length of the transmission line, the IBR penetration level, and critical control parameters, among others. It is shown that by tuning these parameters, the IBR penetration level can be significantly increased. Further, the paper develops a generic and expandable model of the power network with both IBRs and SGs. Further study will focus on transient stability analysis to achieve a holistic understanding of low-inertia power grids with $100 \%$ IBR penetration level with both small- and large-signal stability analysis.

\section{ACKNOWLEDGMENT}

This work was authored in part by the National Renewable Energy Laboratory, operated by Alliance for Sustainable Energy, LLC, for the U.S. Department of Energy (DOE) under Contract No. DE-AC36-08GO28308. This material is based upon work supported by the U.S. Department of Energy's Office of Energy Efficiency and Renewable Energy (EERE) under the Solar Energy Technologies Office Award Number 34224 and 37772. The U.S. Government retains and the publisher, by accepting the article for publication, acknowledges that the U.S. Government retains a nonexclusive, paid-up, irrevocable, worldwide license to publish or reproduce the published form of this work, or allow others to do so, for U.S. Government purposes. The views expressed herein do not necessarily represent the views of the U.S. Department of Energy or the United States Government.

\section{REFERENCES}

[1] U. Markovic, O. Stanojev, P. Aristidou, E. Vrettos, D. S. Callaway and G. Hug, "Understanding Small-Signal Stability of Low-Inertia Systems," IEEE Trans. Power Syst., in press.

[2] P. Kundur, Power System Stability and Control (ser. The EPRI Power System Engineering). Palo Alto, CA, USA: McGraw-Hill, 1994.

[3] Q. Peng, Q. Jiang, Y. Yang, T. Liu, H. Wang and F. Blaabjerg, "On the Stability of Power Electronics-Dominated Systems: Challenges and Potential Solutions," IEEE Trans. Industry Appl., vol. 55, no. 6, pp. 76577670, 2019.

[4] K. Kawabe and K. Tanaka, "Impact of Dynamic Behavior of Photovoltaic Power Generation Systems on Short-Term Voltage Stability," IEEE Trans. Power Syst., vol. 30, no. 6, pp. 3416-3424, 2015.

[5] D. Gautam, V. Vittal and T. Harbour, "Impact of Increased Penetration of DFIG-Based Wind Turbine Generators on Transient and Small Signal Stability of Power Systems," IEEE Trans. Power Syst., vol. 24, no. 3, pp. 1426-1434, 2009.

[6] R. Rosso, X. Wang, M. Liserre, X. Lu and S. Engelken, "Grid-Forming Converters: Control Approaches, Grid-Synchronization, and Future Trends-A Review," IEEE Open Journal of Industry Appl., vol. 2, pp. 93109, 2021, in press.

[7] D. Pattabiraman, J. Tan, V. Gevorgian, A. Hoke, C. Antonio and D. Arakawa, "Impact of Frequency-Watt Control on the Dynamics of a High DER Penetration Power System," in Proc. of IEEE Power \& Energy Society General Meeting (PESGM), pp. 1-5, 2018.

[8] Y. Du, X. Lu, J. Wang, B. Chen, H. Tu, and S. Lukic, "Dynamic Microgrids in Resilient Distribution Systems with Reconfigurable CyberPhysical Networks," IEEE J. Emerg. Sel. Topics Power Electron., pp. 1$1,2020$.

[9] D. Pattabiraman, R. H. Lasseter. and T. M. Jahns, "Comparison of Grid Following and Grid Forming Control for a High Inverter Penetration Power System," in 2018 IEEE Power \& Energy Society General Meeting (PESGM), 2018, pp. 1-5.

[10] D. Pan, X. Ruan, C. Bao, W. Li, and X. Wang, "Capacitor-CurrentFeedback Active Damping With Reduced Computation Delay for Improving Robustness of LCL-Type Grid-Connected Inverter," IEEE Trans. Power Electron., vol. 29, no. 7, pp. 3414-3427, 2014.

[11] N. Pogaku, M. Prodanovic, and T. C. Green, "Modeling, Analysis and Testing of Autonomous Operation of an Inverter-Based Microgrid," IEEE Trans. Power Electron., vol. 22, no. 2, pp. 613-625, 2007.

[12] L. Ding, Y. Men, Y. Du, X. Lu, B. Chen, J. Tan, Y. Lin, "Region-based Stability Analysis of Resilient Distribution Systems with Hybrid Gridforming and Grid-following Inverters," in Proc. of IEEE Energy Conversion Congress and Exposition (ECCE), pp. 3733-3740, 2020.

[13] R. W. Kenyon, A. Hoke, J. Tan and B. Hodge, "Grid-Following Inverters and Synchronous Condensers: A Grid-Forming Pair?," in Proc. of Clemson University Power Systems Conference (PSC), pp. 1-7, 2020. 